J. Korean Math. Soc. 47 (2010), No. 1, pp. 123-134

DOI 10.4134/JKMS.2010.47.1.123

\title{
MORSE INEQUALITIES FOR MANIFOLDS WITH BOUNDARY
}

\author{
Mostafa Esfahani ZadeH
}

\begin{abstract}
The aim of this paper is to provide a proof for a version of the Morse inequalities for manifolds with boundary. Our main results are certainly known to the experts on Morse theory, nevertheless it seems necessary to write down a complete proof for it. Our proof is analytic and is based on the J. Roe account of Witten's approach to Morse Theory.
\end{abstract}

\section{Introduction}

The Morse inequalities is among the most fundamental results in differential topology. For a Morse-Smale function on a closed manifold these inequalities follow from the fact that the homology of the Thom-Smale complex is isomorphic to the singular homology of the manifold, c.f. [7]. In his very influential paper [10], Witten used the Morse function to deform the de Rham complex of the manifold and gave a purely analytic proof for the Morse inequalities. In fact the Thom-Smale complex can be identified with a finite dimensional subcomplex of the deformed de Rham complex. This subcomplex corresponds to the small eigenvalues of the deformed Laplacian. This identification was established by Helffer and Sjöstrand by using methods of the semi-classical analysis. A simple proof of this identification is provided by Bismut-Zhang. For a thorough discussion of this proof and other related matters we refer to [11]. In this paper we will deal with the Morse inequalities when the underlying manifold has non empty boundary.

Let $M$ be a smooth manifold with boundary $\partial M \neq \emptyset$ and let $f: M \rightarrow R$ be a smooth function whose critical points are either isolated nondegenerate located in $M \backslash \partial M$ or located on the boundary such that each connected component of the boundary is a non degenerate critical sub manifold of index 0 or 1 in the sense of Bott [4]. More precisely let $\partial M=N_{+} \sqcup N_{-}$be a disjoint union of closed manifolds so that $f(u, y)=\frac{1}{2} u^{2}$ in a collar neighborhood $[0,1) \times N_{+}$ while $f(u, y)=-\frac{1}{2} u^{2}$ in a collar neighborhood $[0,1) \times N_{-}$. The Bott index of

Received March 20, 2008; Revised February 2, 2009.

2000 Mathematics Subject Classification. Primary 58E05; Secondary 35J25.

Key words and phrases. Morse inequalities, Witten's Laplacian, Dirichlet and Neumman boundary conditions. 
each component of $N_{+}$is 0 while each component of $N_{-}$has Bott index 1 . In both case $f$ satisfies the Neumann condition

$$
\frac{\partial f}{\partial u}(0, y)=0 \text {. }
$$

Let $N_{+}=N_{a+} \sqcup N_{r+}$ and $N_{-}=N_{a-} \sqcup N_{r-}$ be disjoint union of closed manifolds. The subscripts " $a$ " and " $r$ " refer respectively to absolute and relative boundary condition that we explain in below. In sequel we consider a Riemannian metric on $M$ which is Euclidian around non degenerate critical points with respect to coordinates provided by Morse lemma. Moreover in the collar neighborhood $(1,0] \times \partial M$ it is assumed to take the product form $g=d^{2} u+g_{0}$ where $g_{0}$ is a Riemannian metric on $\partial M$. So the flow of $\nabla f$, the gradient of $f$ with respect to this metric, is defined for $t \in \mathbb{R}$ and limit points of each integral curve is either a non degenerate critical point in the interior of $M$ or a point on $\partial M_{i}$. Put

$$
\begin{aligned}
\beta_{k} & =\operatorname{dim} H_{d r}^{k}\left(M, N_{r}\right), \\
\gamma_{k} & =\operatorname{dim} H_{d r}^{k}\left(N_{r-}\right), \\
\eta_{k} & =\operatorname{dim} H_{d r}^{k}\left(N_{a+}\right) .
\end{aligned}
$$

We denote by $c_{k}$ the number of nondegenerate critical points of Morse index $k$. The aim of this note is to provide a proof for the following theorem by using the Witten approach to Morse theory [10].

Theorem 1. The following inequalities hold for $0 \leq k \leq n$

$$
\mu_{k}-\mu_{k-1}+\cdots+(-1)^{k} \mu_{0} \geq \beta_{k}-\beta_{k-1}+\cdots+(-1)^{k} \beta_{0},
$$

where

$$
\mu_{k}=c_{k}+\eta_{k}+\gamma_{k-1} .
$$

The equality holds for $k=n$.

Notice that the equality for $k=n$ reads

$$
\sum_{k=0}^{n}(-1)^{n-k} c_{k}=\chi\left(M, N_{r}\right)+\chi\left(N_{a+}\right)-\chi\left(N_{r-}\right) .
$$

As a special case let $\operatorname{dim} M$ be an odd integer and let $f$ be a Morse function on $M$ such that $\partial M=N_{a+}$. Then the above relation reads

$$
\sum_{k=0}^{n}(-1)^{n-k} c_{k}=\chi(M)+\chi(\partial M) .
$$

Now consider the Morse function $-f$ for which $\partial M=N_{a-}$, we get the following relation

$$
-\sum_{k=0}^{n}(-1)^{n-k} c_{k}=\chi(M)
$$


Comparing these two relations one gets the following relation proving that the parity of the Euler character is a cobordism invariant.

$$
\chi(\partial M)=-2 \chi(M) .
$$

For example the Euler characteristic of a manifold which is the boundary of a contractible odd dimensional manifolds, e.g. $S^{2 p}$ equals -2 . A similar argument gives the relation $\chi(\partial M)=2 \chi(M, \partial M)$. Of course these relations can be obtained by homotopy theory and Poincare duality.

\section{Analytical properties of deformed Laplacian with boundary conditions}

To give a proof for Theorem 1, we begin by studying the Laplacian operator, and its deformation, on the de Rham complex endowed with boundary conditions. Let $\omega$ be a differential $k$-form on $M$. In collar neighborhood $U$ of $\partial M$ it takes the following form

$$
\omega_{\mid U}=\omega_{1}(u, y)+d u \wedge \omega_{2}(u, y),
$$

where $\omega_{i}$ 's are $u$-depending differential forms on $\partial M$. Differential form $\omega$ satisfies the relative boundary condition $B_{r}$ if

$$
\omega_{1}(0, y)=0 \quad \text { and } \quad \frac{\partial \omega_{2}}{\partial u}(0, y)=0 .
$$

Differential form $\omega$ satisfies the absolute boundary condition $B_{a}$ if

$$
\frac{\partial \omega_{1}}{\partial u}(0, y)=0 \quad \text { and } \quad \omega_{2}(0, y)=0 .
$$

Clearly $\omega$ satisfies $B_{r}$ if and only if $* \omega$ satisfies $B_{a}$, where $*$ is a locally defined Hodge star operator with respect to a local orientation. From now on we impose the relative boundary condition on $N_{r}$ and the absolute boundary condition on $N_{a}$ and denote this setting of boundary condition by $B$. By performing the completion of the set of smooth differential forms satisfying the boundary conditions $B$ with respect to appropriate Sobolev norm, we obtain the Sobolev spaces $W^{l}\left(M, \Lambda^{*} T^{*} M ; B\right)$. The inclusion $W^{l}\left(M, \Lambda^{*} T^{*} M ; B\right) \hookrightarrow W^{l}\left(M, \Lambda^{*} T^{*} M\right)$ is an isometry with closed image. So all classical theorems in the theory of the Sobolev spaces, e.g. Sobolev embedding theorem, Rellich's theorem and the elliptic estimate hold also in this context.

Let $\eta$ be an another differential form on $M$ taking the form $\eta=\eta_{1}+d u \wedge \eta_{2}$ in collar neighborhood of $\partial M$. The Green formula for $D:=d+\delta$ (c.f. $[3$, page 24]) takes the following form in our context

$$
\langle D \omega, \eta\rangle-\langle\omega, D \eta\rangle=-\int_{\partial M}\left\langle\omega_{1}, \eta_{2}\right\rangle+\int_{\partial M}\left\langle\omega_{2}, \eta_{1}\right\rangle .
$$

So the operator $D$ is formally self adjoint provided $\omega$ and $\eta$ satisfy both the relative or the absolute boundary conditions. Consequently $D$ is a formally self 
adjoint operator on $\Omega^{*}(M, B)$ and Laplacian operator $\triangle:=D^{2}$ is a formally positive second order elliptic operator on $\Omega^{*}(M, B)$ and

$$
\operatorname{ker} \triangle=\operatorname{ker} D \text {. }
$$

The heat kernel of $e^{-t \triangle}$ may be constructed by means of heat kernels of boundary problems on half cylinder $\mathbb{R}^{\geq 0} \times \partial M$ and heat kernel of Laplacian on closed manifold $M \sqcup_{\partial M} M$ (see [1, page 55]). Since the function $f$ satisfies the Neumann condition (1.1), the multiplication by $e^{ \pm s f}$ preserves the boundary condition $B$. Here, and so on, $s$ is a non negative real number. Let $d_{s}:=e^{-s f} d e^{s f}$ and $\delta_{s}=e^{s f} \delta e^{-s f}$ and put

$$
D_{s}:=d_{s}+\delta_{s} \quad \text { and } \quad \triangle_{s}:=D_{s}^{2} .
$$

As in above $D_{s}$ is formally self adjoint, so the Witten Laplacian $\triangle_{s}$ is a formally positive elliptic differential form and

$$
\operatorname{ker} \triangle_{s}=\operatorname{ker} D_{s}=\operatorname{ker} d_{s} \cap \operatorname{ker} \delta_{s} .
$$

The Hessian of the function $f$ is the following 2-tensor

$$
H(X, Y):=X .(Y . f)-\left(\nabla_{X} Y\right) . f .
$$

Let $\left\{e_{i}\right\}$ be a local orthonormal basis for $T M$ and $\alpha \in \Lambda^{k} T^{*} M$. Put $L_{e_{i}}(\alpha):=$ $e^{i} . \alpha$ and $R_{e_{i}}(\alpha):=(-1)^{k} \alpha \cdot e^{i}$, where dot "." denotes the Clifford multiplication. More explicitly $\left.L_{e_{i}}(\alpha)=e_{i} \wedge \alpha+e_{i}\right\lrcorner \alpha$ and $\left.R_{e_{i}}(\alpha)=e_{i} \wedge \alpha-e_{i}\right\lrcorner \alpha$, where $\lrcorner$ denotes the interior multiplication, cf. [8, page 49]. The following relation defines a smooth section of $\operatorname{End}\left(\Lambda^{*} \mathrm{~T}^{*} \mathrm{M}\right)$ which is independent of the orthonormal basis

$$
\mathrm{H}=\sum_{i, j} H\left(e_{i}, e_{j}\right) L_{e_{i}} R_{e_{j}} .
$$

With these notation we have the following relation [8, Proposition 9.17]

$$
\triangle_{s}^{2}=\triangle^{2}+s \mathrm{H}+s^{2}|d f|^{2} .
$$

Notice that the operator $\mathrm{H}$ preserves clearly the boundary condition $B$. Moreover, due to the relation (1.1) the multiplication operator $|d f|$ preserve this boundary condition too. Using (2.5) and the Duhamel formula (see, e.g. [2]), one can construct the heat operator $e^{-t \triangle_{s}}$ by means of $e^{-t \triangle}$. The heat operator is a smoothing operator on $L^{2}\left(M, \Lambda^{*} T^{*} M\right)$, so the Sobolev embedding theorem implies that it is a compact self adjoint operator on this Hilbert space. So, for each $0 \leq k \leq n$, the Hilbert space $L^{2}\left(M, \wedge{ }^{k} T^{*} M\right)$ may be decomposed to orthogonal sum of eigenspaces of $\triangle_{s}^{k}$.

$$
L^{2}\left(M, \wedge^{k} T^{*} M\right)=\overline{\oplus_{\lambda_{s} \geq 0} E_{\lambda_{s}}^{k}} .
$$

The following sequence is exact for $0 \neq \lambda_{s} \in \operatorname{Spec}\left(\triangle_{s}\right)$

$$
0 \rightarrow E_{\lambda_{s}}^{0} \stackrel{d_{s}}{\rightarrow} E_{\lambda_{s}}^{1} \stackrel{d_{s}}{\rightarrow} \cdots \stackrel{d_{s}}{\rightarrow} E_{\lambda_{s}}^{n-1} \stackrel{d_{s}}{\rightarrow} E_{\lambda_{s}}^{n} \rightarrow 0 .
$$

Notice that as a part of assertion, the exterior differential preserves the boundary conditions when it is restricted to eigenspaces of $\triangle_{s}$. We refer to $[5$, page 
49] for a proof of this assertion. Since $d_{s}$ preserves ker $\triangle$ the above assertion implies that the cohomology of the deformed de Rham complex

$$
0 \rightarrow \Omega^{0}(M, B) \stackrel{d_{s}}{\rightarrow} \Omega^{1}(M, B) \stackrel{d_{s}}{\rightarrow} \cdots \stackrel{d_{s}}{\rightarrow} \Omega^{n}(M, B) \rightarrow 0,
$$

is isomorphic to ker $\triangle_{s}$ and the isomorphism is induced from equality (2.4). Moreover the multiplication by $e^{s f}$ provides an isomorphism between this complex and the ordinary de Rham complex corresponding to $s=0$. The cohomology of de Rham complex is the relative cohomology group $H_{d r}^{*}\left(M . N_{r}\right)$. So, as an immediate consequence of the above discussion we get the following relation

$$
\beta_{k}=\operatorname{dim} \operatorname{ker} \triangle_{s}^{k} \text {. }
$$

The basic analytical tool in the next section is the finite propagation speed property of wave equation that we are going to explain. Let $\omega$ and $\omega^{\prime}$ be two differential $p$-forms on $M$ which satisfy the boundary condition $B$. There is a unique smooth $t$-depending differential $p$-form $\omega_{t}$ satisfying the boundary condition $B$ and the initial conditions $\omega_{0}=\omega$ and $\left(\partial_{t} \omega_{t}\right)_{t=0}=\omega^{\prime}$ such that $\omega_{t}$ is a solution of the wave equation

$$
\frac{\partial^{2} \omega_{t}}{\partial^{2} t}+D_{s}^{2} \omega_{t}=0
$$

For the proof of the above facts and the following lemma we refer to [9, pages 145-148].

Lemma 2 (unite propagation speed property of wave equation). With the above notation if $\omega$ is compactly supported then $\omega_{t}$ is supported inside the distance $|t|$ of $\operatorname{supp}(\omega)$.

\section{Derivation of the Morse inequalities}

Let $\phi$ be a non negative rapidly decreasing function on $\mathbb{R}^{\geq 0}$ satisfying $\phi(0)=$ 1. The operator $\phi\left(\triangle_{s}^{k}\right)$ being a smoothing operator, is of trace class, so we can define $\nu_{k}(s):=\operatorname{Tr} \phi\left(\triangle_{s}^{k}\right)$. The following proposition is a generalized version of the Proposition 14.3 of [8] in the presence of the boundary. We prove this theorem by using the spectral resolution provided by eigenvectors of $\triangle_{s}$.

Proposition 3. The following inequalities hold for $0 \leq k \leq n$

$$
\nu_{k}(s)-\nu_{k-1}(s)+\cdots+(-1)^{k} \nu_{0}(s) \geq \beta_{k}-\beta_{k-1}+\cdots+(-1)^{k} \beta_{0} .
$$

and equality holds for $k=n$.

Proof. Consider the following exact sequence coming from (2.7)

$$
0 \rightarrow E_{\lambda_{s}}^{0} \stackrel{d_{s}}{\rightarrow} E_{\lambda_{s}}^{1} \stackrel{d_{s}}{\rightarrow} \cdots \stackrel{d_{s}}{\rightarrow} E_{\lambda_{s}}^{k} \stackrel{d_{s}}{\rightarrow} \operatorname{Im} d_{s}^{k} \rightarrow 0 .
$$

Obviously $\phi\left(\triangle_{s}\right)$ restricts to a linear operator on $\operatorname{Im} d_{s}^{k}$. The trace of this restriction, denoted by $r\left(\phi, \lambda_{s}, k\right)$, is non negative for $0 \leq k \leq n$ and is zero 
for $k=n$. Since $\triangle_{s \mid E_{\lambda_{s}}}=\lambda_{s}$ id, the trace of the restriction of $\phi\left(\triangle_{s}\right)$ to $E_{\lambda_{s}}^{j}$ equals $\phi\left(\lambda_{s}\right) \cdot \operatorname{dim} E_{\lambda_{s}}^{j}$. So

$$
r\left(\phi, \lambda_{s}, k\right)-\operatorname{tr} \phi\left(\triangle_{\lambda_{s} \mid E_{\lambda_{s}}^{k}}^{k}\right)+\operatorname{tr} \phi\left(\triangle_{\lambda_{s} \mid E_{\lambda_{s}}^{k-1}}^{k-1}\right)-\cdots+(-1)^{k} \operatorname{tr} \phi\left(\triangle_{\lambda_{s} \mid E_{\lambda_{s}}^{0}}^{0}\right)=0 .
$$

By summation over all $\lambda_{s} \neq 0$ and using the fact that $\phi(0)=1$ and (2.8) we get

$\sum_{\lambda_{s} \neq 0} r\left(\phi, \lambda_{s}, k\right)-\left(\mu_{k}(s)-\beta_{k}\right)+\left(\mu_{k-1}(s)-\beta_{k-1}\right)-\cdots+(-1)^{k}\left(\mu_{0}(s)-\beta_{0}\right)=0$.

These inequalities prove the assertion of the proposition because for $0 \leq k \leq n$ one has $r\left(\phi, \lambda_{s}, k\right) \geq 0$ and equality holds for $k=n$.

With above proposition, to prove the Morse inequalities of Theorem 1 we have to study the traces $\nu_{k}(s)=\operatorname{Tr} \phi\left(\triangle_{s}^{k}\right)$ when $s$ goes to infinity. For a small positive number $\rho<1 / 4$, let $M_{\rho}$ denote the disjoint union of $\rho$-neighborhoods of critical points and the $\rho$-collar neighborhood $(\rho, 0] \times \partial M$ of the boundary. The positive number $\rho$ is so small that each connected component of $M_{4} \rho$ contains only one critical point or one connected component of boundary $\partial M$. We recall that $\phi\left(\triangle_{s}^{k}\right)$ is a smoothing operator with smooth kernel $K\left(s, x, x^{\prime}\right) \in$ $\wedge^{k} T_{x} M \otimes \wedge{ }^{k} T_{x^{\prime}} M$.

Proposition 4. Let $\phi$ be a rapidly decreasing even function such that the fourier transform of function $\psi$ defined by $\psi(t):=\phi\left(t^{2}\right)$ is supported in $(-\rho, \rho)$. When $s$ goes toward infinity, the kernel $K(s, x, x)$ of $\phi\left(\triangle_{s}^{k}\right)$ goes uniformly to 0 for $x$ in $M \backslash M_{2 \rho}$.

Proof. In the complement of $M_{4 \rho}$ one has $|d f| \geq c$ for some positive constant c. So, using the relation (2.5), one get the following estimate

$$
\left\langle D_{s}^{2} \omega, \omega\right\rangle_{2} \geq \frac{1}{2} c s^{2}\|\omega\|_{2}^{2} \quad \text { if } \quad \operatorname{supp}(\omega) \subset M_{4 \rho} .
$$

Using this inequality, the finite propagation speed property of Lemma 2 and classical theorem of Sobolev spaces $W^{k}\left(M, \Lambda T^{*} M ; B\right)$, the proof of the Proposition 14.6 in [8] goes over verbatim and prove this proposition.

Let $\beta$ be a smooth function on $M$ which is supported in $M_{3 \rho}$ and is equal to 1 on $M_{2 \rho}$. The above lemma shows that

$$
\lim _{s \rightarrow \infty} \operatorname{Tr} \phi\left(\triangle_{s}^{k}\right)=\lim _{s \rightarrow \infty} \operatorname{Tr} \bar{\beta} \phi\left(\triangle_{s}^{k}\right),
$$

where $\bar{\beta}$ is the pointwise multiplication by $\beta$. So, the next step is to study the asymptotic behavior of $\operatorname{Tr} \bar{\beta} \phi\left(\triangle_{s}^{k}\right)$ when $s$ goes to infinity. From now on we consider $M_{4}$ either with coordinates given by Morse lemma around the non degenerate critical points, or by collar coordinates $(u, y)$ in $(4 \rho, 0] \times \partial M$. We assume that the Riemannian structure is Euclidian around critical points and is of product form $d u^{2}+g_{0}$ in the collar neighborhood. Using these coordinates we can identify the differential form supported in $M_{4 \rho}$ with differential forms 
supported either in the $4 \rho$-neighborhood of origin in $\mathbb{R}^{n}$ or in product space $(4 \rho, 0] \times \partial M$. In the neighborhood of a critical point $x_{0} \in M$ with Morse index $r$, the function $f$ takes the following form

$$
f\left(x_{1}, \ldots, x_{n}\right)=f\left(x_{0}\right)-\frac{1}{2} x_{1}^{2}-\cdots-\frac{1}{2} x_{r}^{2}+\cdots+\frac{1}{2} x_{n}^{2} .
$$

So the deformed Laplacian $\triangle_{s}^{k}$ given by relation $(2.5)$ coincides with the following operator acting on $\Omega^{k}\left(\mathbb{R}^{n}\right)$

$$
L_{s}^{k}=-\sum_{j=1}^{n} \frac{\partial^{2}}{\partial^{2} x_{j}}+s^{2} x_{j}^{2}+s \epsilon_{j} Z_{j} .
$$

Here $\epsilon_{j}= \pm 1$ is the sign of the coefficient of $x_{j}^{2}$ in the expression (3.5). Moreover $Z_{j}:=\left[d x_{j} \wedge ., d x_{j}\llcorner\right.$.$] . In the collar neighborhood [0,1) \times N_{+}$we have $f(u, y)=$ $\frac{1}{2} u^{2}$. So the expression (2.5) for $\triangle_{s}^{k}$ gives the following operator acting on $\oplus_{\epsilon=0,1} \Omega^{\epsilon}([0,1), B) \otimes \Omega^{k-\epsilon}\left(N_{+}\right)$

$$
L_{s}^{k}=\left(A_{s}^{0}+\triangle_{N_{+}}^{k}\right) \oplus\left(A_{s}^{1}+\triangle_{N_{+}}^{k-1}\right),
$$

where

$$
A_{s}^{\epsilon}=-\frac{\partial^{2}}{\partial u^{2}}+s^{2} u^{2}+(-1)^{\epsilon+1} s .
$$

In the collar neighborhood $[0,1) \times N_{-}$we have $f(u, y)=-\frac{1}{2} u^{2}$. So the expression (2.5) for $\triangle_{s}^{k}$ gives the following operator acting on $\oplus_{\epsilon=0,1} \Omega^{\epsilon}([0,1), B) \otimes$ $\Omega^{k-\epsilon}\left(N_{-}\right)$

$$
L_{s}^{k}=\left(A_{s}^{0}+\triangle_{N_{-}}^{k}\right) \oplus\left(A_{s}^{1}+\triangle_{N_{-}}^{k-1}\right)
$$

where

$$
A_{s}^{\epsilon}=-\frac{\partial^{2}}{\partial u^{2}}+s^{2} u^{2}+(-1)^{\epsilon} s
$$

In above discussion $\triangle_{N_{+}}^{k-1}$ and $\triangle_{N_{-}}^{k-1}$ are, respectively, the Laplacian operators on $N_{+}$and $N_{-}$.

Proposition 5. Let $L_{s}^{k}$ denote any one of operators given by (3.6), (3.7) or (3.8) and let $\bar{\beta}$ be the corresponding operator defined in above. The following equality holds

$$
\left.\operatorname{Tr} \bar{\beta} \phi\left(\triangle_{s}^{k}\right)\right)=\operatorname{Tr} \bar{\beta} \phi\left(L_{s}^{k}\right) .
$$

Here $\phi\left(\triangle_{s}^{k}\right)$ and $\phi\left(L_{s}^{k}\right)$ are bounded operator on different $L^{2}$-Hilbert spaces.

Proof. Clearly $\triangle_{s}=D_{s}^{2}$ and $L_{s}=A_{s}^{2}$ where $A_{s}$ is a differential operator which is equal to $D_{s}$ in $M_{4 \rho}$. Define the function $\psi$ by $\psi(t):=\phi\left(t^{2}\right)$. To prove the proposition it suffices to prove the following equality,

$$
\psi\left(D_{s}\right) \omega=\psi\left(A_{s}\right) \omega
$$

provided that $\omega$ is supported in $M_{3 \rho}$. For this purpose notice that the relations $\omega_{t}:=\cos \left(t D_{s}\right) \omega$ and $\omega_{t}^{\prime}:=\cos \left(t A_{s}\right) \omega$ are smooth solutions of wave equation 
with the same initial conditions. Therefore the unite propagation speed property of Lemma 2 implies the equality $\omega_{t}=\omega_{t}^{\prime}$ for $|t|<\rho$. Using the fact that $\hat{\psi}$ is an even function supported in $[-\rho, \rho]$ we get

$$
\begin{aligned}
\psi\left(D_{s}\right) \omega & =\frac{1}{2 \pi} \int_{-\rho}^{\rho} \hat{\psi}(t) e^{i t D_{s}} \omega d t \\
& =\frac{1}{\pi} \int_{0}^{\rho} \hat{\psi}(t) \cos \left(t D_{s}\right) \omega d t \\
& =\frac{1}{\pi} \int_{0}^{\rho} \hat{\psi}(t) \cos \left(t L_{s}\right) \omega d t \\
& =\psi\left(A_{s}\right) \omega .
\end{aligned}
$$

This completes the proof of the proposition.

In view of the above proposition, for computing $\nu_{k}(s)$ when $s$ goes toward $\infty$, it suffices to compute $\operatorname{Tr}\left(\bar{\beta} L_{s}^{k}\right)$, at $s=\infty$, where $L_{s}^{k}$ is given by relations (3.6), (3.7), and (3.8).

Concerning the operator $L_{s}^{k}$ given by (3.6), the following relation holds, cf. [8, Lemma 14.11]

$$
\lim _{s \rightarrow \infty} \operatorname{Tr}\left(\bar{\beta} \phi\left(L_{s}^{k}\right)\right)= \begin{cases}0, & k \neq r \\ 1, & k=r .\end{cases}
$$

Now we are going to compute $\lim _{s \rightarrow 0} \operatorname{Tr}\left(\bar{\beta} \phi\left(L_{s}^{k}\right)\right)$ for $L_{s}^{k}$ of relations (3.7) and (3.8). For this purpose, we summarize some basic properties of the one dimensional harmonic oscillator operator (see [8, Chapter 9])

$$
-\frac{\partial^{2}}{\partial^{2} u}+s^{2} u^{2}: \mathcal{S}(\mathbb{R}) \rightarrow \mathcal{S}(\mathbb{R})
$$

where $\mathcal{S}(\mathbb{R})$ denotes the space of smooth rapidly decreasing functions on $\mathbb{R}$. The eigenvalues of this operator are $(2 p+1) s$ for $p=0,1,2, \ldots$ and each eigenvalue has multiplicity one. The corresponding eigenfunctions have the following form

$$
\vartheta_{p}(s, u)=\left(2^{p} s^{p+1} p !\right)^{-\frac{1}{2}}\left(-\frac{d}{d u}+s u\right)^{p} e^{-\frac{s u^{2}}{2}} .
$$

In particular these eigenfunctions satisfy the Dirichlet boundary condition when $p$ is odd and the Neumann condition when $p$ is even. Another basic property is related to $\vartheta_{0}$. If $\beta$ is a rapidly decreasing continuous function defined around $0 \in \mathbb{R}$, then

$$
\lim _{s \rightarrow \infty}\left\langle\left(\beta(\cdot) \vartheta_{0}(s, \cdot)\right), \vartheta_{0}(s, \cdot)\right\rangle=\beta(0) .
$$

Obviously the harmonic oscillator operator may be considered as an operator on the Schwartz space $\mathcal{S}(\mathbb{R}) d u$ of differential 1-forms. The eigenvalues of this operator are the same and the eigenvectors are $\vartheta_{p}(s, u) d u$.

In what follows we will need to consider a sequence $\phi_{m} ; m \in \mathbb{N}$ of rapidly decreasing non negative even functions on $\mathbb{R}$ satisfying $\phi_{m}(0)=1$ such that 
the Fourier transforms $\hat{\psi}_{m}$ is supported in $(-\rho, \rho)$ where $\psi_{m}(t)=\phi_{m}\left(t^{2}\right)$. Moreover the sequence $\phi_{m}$ converges uniformly to zero outside the compact neighborhoods of $0 \in \mathbb{R}$.

3.0.1. Cylindrical operators arising from $\boldsymbol{N}_{+}$. On the product space $\mathbb{R}^{\geq 0} \times N_{+}$ we consider the deformed Laplacian operator $L_{s}^{k}=A_{s}^{\epsilon}+\triangle_{N_{+}}$given by (3.7) and acting on $\Omega^{\epsilon}\left([0,1), B_{r}\right) \otimes \Omega^{k-\epsilon}(\partial M)$. Here

$$
A_{s}^{\epsilon}=-\frac{\partial^{2}}{\partial u^{2}}+s^{2} u^{2}+(-1)^{\epsilon+1} s .
$$

Let $\left\{\psi_{\lambda_{k}}^{k}\right\}_{\lambda_{k}}$ be a spectral resolution for $\triangle_{s}^{k}$. So the eigenvectors of the restriction of $L_{s}^{k}$ to $\mathbb{R}^{\geq 0} \times N_{r+}$ with respect to boundary condition (2.1) are

$$
\vartheta_{2 l+1} \otimes \psi_{\lambda_{k}}^{k} \quad ; \quad \vartheta_{2 l} d u \otimes \psi_{\lambda_{k-1}}^{k-1}, \quad l=0,1,2, \ldots
$$

So

$$
\begin{aligned}
\operatorname{Tr} \bar{\beta} \phi_{m}\left(L_{s \mid \mathbb{R} \geq 0 \times N_{r+}}^{k}\right)= & \sum_{l, \lambda_{k}} \phi_{m}\left(s+4 l s+\lambda_{k}\right)\left\langle\beta(\cdot) \vartheta_{2 l+1}(s, \cdot), \vartheta_{2 l+1}(s, \cdot)\right\rangle \\
& +\sum_{l, \lambda_{k-1}} \phi_{m}\left(2 s+4 l s+\lambda_{k-1}\right)\left\langle\beta(\cdot) \vartheta_{2 l}(s, \cdot), \vartheta_{2 l}(s, \cdot)\right\rangle .
\end{aligned}
$$

The function $\phi_{m}$ is rapidly decreasing at infinity and the arguments of $\phi_{m}$ appearing in above formula go to infinity when $s$ do, so

$$
\lim _{s \rightarrow \infty} \operatorname{Tr} \bar{\beta} \phi_{m}\left(L_{s \mid \mathbb{R} \geq 0 \times N_{r+}}^{k}\right)=0 .
$$

Now we consider the case of $L_{s \mid \mathbb{R} \geq 0 \times N_{a+}}^{k}$, given by (3.8), with respect to absolute boundary conditions (2.2). According to above discussion, the eigenvectors of this operators are

$$
\vartheta_{2 l} \otimes \psi_{\lambda_{k}}^{k} \quad ; \quad \vartheta_{2 l+1} d u \otimes \psi_{\lambda_{k-1}}^{k-1}, \quad l=0,1,2, \ldots
$$

So we have

$$
\begin{aligned}
\operatorname{Tr} \bar{\beta} \phi_{m}\left(L_{s \mid \mathbb{R} \geq 0 \times N_{r+}}^{k}\right)= & \sum_{l, \lambda_{k}} \phi_{m}\left(4 l s+\lambda_{k}\right)\left\langle\beta(\cdot) \vartheta_{2 l}(s, \cdot), \vartheta_{2 l}(s, \cdot)\right\rangle \\
& +\sum_{l, \lambda_{k-1}} \phi_{m}\left(3 s+4 l s+\lambda_{k-1}\right)\left\langle\beta(\cdot) \vartheta_{2 l+1}(s, \cdot), \vartheta_{2 l+1}(s, \cdot)\right\rangle .
\end{aligned}
$$

The argument of $\phi_{m}$ is second summation appearing in above equalities go all toward infinity when $s$ do, so this sum has no contribution when $s$ goes to infinity. The arguments of $\phi_{m}$ in the first summation, except those corresponding to $l=\lambda_{k}=0$, have no contribution because $\phi_{m}$ 's are rapidly decreasing at infinity and because the sequence $\left\{\phi_{m}\right\}_{m}$ converges uniformly to zero out of compact neighborhoods of $0 \in \mathbb{R}$. Therefore

$$
\lim _{s, m \rightarrow \infty} \operatorname{Tr} \bar{\beta} \phi_{m}\left(L_{s \mid \mathbb{R} \geq 0 \times N_{a+}}^{k}\right)=\lim _{s, m \rightarrow \infty} \sum_{\lambda_{k}=0} \phi_{m}(0)\left\langle\beta(\cdot) \vartheta_{0}(s, \cdot), \vartheta_{0}(s, \cdot)\right\rangle .
$$


Now using the relation (3.10) and using the Hodge theory on $N_{a+}$ we conclude

$$
\lim _{s \rightarrow \infty} \operatorname{Tr} \bar{\beta} \phi_{m}\left(L_{s \mid \mathbb{R} \geq 0 \times N_{a+}}^{k}\right)=\eta_{k}
$$

3.0.2. Cylindrical operators arising from $\boldsymbol{N}_{-}$. On the product space $\mathbb{R}^{\geq 0} \times N_{-}$ we consider the deformed Laplacian operator $L_{s}=A_{s}^{\epsilon}+\triangle_{\partial M}^{j}$ given by (3.8) and acting on $\Omega^{\epsilon}\left([0,1), B_{r}\right) \otimes \Omega^{j}(\partial M)$. Here

$$
A_{s}^{\epsilon}=-\frac{\partial^{2}}{\partial u^{2}}+s^{2} u^{2}+(-1)^{\epsilon} s .
$$

The eigenvectors of $L_{s}^{k}$ with respect to boundary condition (2.1) are

$$
\vartheta_{2 l+1} \otimes \psi_{\lambda_{k}}^{k} \quad ; \quad \vartheta_{2 l} d u \otimes \psi_{\lambda_{k-1}}^{k-1}, \quad l=0,1,2, \ldots
$$

therefore

$$
\begin{aligned}
\operatorname{Tr} \bar{\beta} \phi_{m}\left(L_{s \mid \mathbb{R} \geq 0 \times N_{r+}}^{k}\right)= & \sum_{l, \lambda_{k}} \phi_{m}\left(3 s+4 l s+\lambda_{k}\right)\left\langle\beta(\cdot) \vartheta_{2 l+1}(s, \cdot), \vartheta_{2 l+1}(s, \cdot)\right\rangle \\
& +\sum_{l, \lambda_{k-1}} \phi_{m}\left(4 l s+\lambda_{k-1}\right)\left\langle\beta(\cdot) \vartheta_{2 l}(s, \cdot), \vartheta_{2 l}(s, \cdot)\right\rangle .
\end{aligned}
$$

All arguments of $\phi_{m}$ in the first summation go to infinity when $s$ do, so this summation has no non-vanishing contribution at $s=\infty$. The sequence $\phi_{m}$ converges uniformly to zero out of compact neighborhoods of $0 \in \mathbb{R}$. So in the second summation, only the terms corresponding to $l=\lambda_{k-1}=0$ may have non vanishing contribution when $s$ and $m$ go to infinity. Therefore using relation $(3.10)$

$$
\lim _{s, m \rightarrow \infty} \operatorname{Tr} \bar{\beta} \phi_{m}\left(L_{s \mid \mathbb{R} \geq 0 \times N_{r-}}^{k}\right)=\lim _{s, m \rightarrow \infty} \sum_{\lambda_{k-1}=0} \phi_{m}(0)\left\langle\beta(\cdot) \vartheta_{0}(s, \cdot), \vartheta_{0}(s, \cdot)\right\rangle
$$

A similar discussion gives the following relation

$$
\left.\lim _{m, s \rightarrow \infty} \operatorname{Tr} \bar{\beta} \phi_{m}\left(L_{s \mid \mathbb{R} \geq 0 \times N_{a-}}^{k}\right)\right)=0 .
$$

Now we are ready to give the proof of Theorem 1 .

Proof of Theorem 1. Put $\mu_{k}(m, s):=\operatorname{Tr} \phi_{m}\left(L_{s}^{k}\right)$. Let $\beta$ be a continuous function supported in small neighborhoods of critical points of $f$ and in collar neighborhood $(1,0] \times \partial M$ of boundary. The value of this function on critical points and on boundary is assumed to be 1 . Moreover in collar neighborhood $(1,0] \times \partial M$ it is assumed to be a function of $u$. We denote by $\bar{\beta}$ the pointwise multiplication of differential forms by $\beta$. From relation (3.4) we get

$$
\lim _{s \rightarrow \infty} \nu_{k}(m, s)=\lim _{s \rightarrow \infty} \operatorname{Tr} \bar{\beta} \phi_{m}\left(\triangle_{s}^{k}\right) .
$$

Proposition 5 implies

$$
\operatorname{Tr} \bar{\beta} \phi_{m}\left(\triangle_{s}^{k}\right)=\sum \operatorname{Tr} \bar{\beta} \phi_{m}\left(L_{s}^{k}\right)
$$


where the sum is taken over connected component of $M_{4 \rho}$. Now relations (3.9), (3.12), (3.13), (3.15), and (3.16) together imply

$$
\lim _{s, m \rightarrow \infty} \sum \operatorname{Tr} \bar{\beta} \phi_{m}\left(L_{s}^{k}\right)=c_{k}+\eta_{k}+\gamma_{k-1} .
$$

This relation with analytic Morse inequalities given in Proposition 3 give the desired results of the theorem.

Remark 1. At the beginning of the paper we assumed that the Morse function $f: M \rightarrow \mathbb{R}$ takes the form $f(u, y)= \pm \frac{1}{2} u^{2}$ in the collar neighborhood $[0,1) \times$ $\partial M$. This condition may be weakened and we could consider smooth functions which are Morse both in the interior and on the boundary of $M$. The restriction of such a function, $f: M \rightarrow \mathbb{R}$, to the boundary is a Morse function too and the inductive proof of the Morse lemma (see [6]) shows that the Morse functions $f$ takes the following form with respect to a local coordinates $u, y_{1}, \ldots, y_{n-1}$

$$
f(u, y)=f\left(x_{0}\right) \pm \frac{1}{2} u^{2} \pm \frac{1}{2} y_{1}^{2} \pm \cdots \pm \frac{1}{2} y_{n-1}^{2} .
$$

Here $\left(y_{1}, \ldots, y_{n-1}\right)$ is a local coordinates system for $\partial M$ around the critical point $x_{0} \in \partial M$. For a connected component $N$ of the boundary $\partial M$, let $c_{k, k^{\prime}}(N)$ denote the number of those critical points $x_{0} \in N$ of $f$ such that $\operatorname{ind}_{x_{0}} f=k$ and $\operatorname{ind}_{x_{0}} f_{N}=k^{\prime}$. As before let $c_{k}$ denote the number of critical points of $f$ with Morse index $k$ which are in the interior of $M$. Put $\mu_{k}:=$ $c_{k}+c_{k, k}\left(N_{a}\right)+c_{k, k-1}\left(N_{r}\right)$ and $\beta_{k}=\operatorname{dim} H_{d r}\left(M, N_{r}\right)$. The above discussion about cylindrical operators and boundary properties of eigenfunctions of the harmonic oscillator can be applied to the operator $L_{s}^{k}$ appeared in relation (3.9) to deduce the following Morse inequalities

$$
\mu_{k}-\mu_{k-1}+\cdots+(-1)^{k} \mu_{0} \geq \beta_{k}-\beta_{k-1}+\cdots+(-1)^{k} \beta_{0} .
$$

As before the equality holds for $k=n$.

\section{References}

[1] M. F. Atiyah, V. K. Patodi, and I. M. Singer, Spectral asymmetry and Riemannian geometry. I, Math. Proc. Cambridge Philos. Soc. 77 (1975), 43-69.

[2] N. Berline, E. Getzler, and M. Vergne, Heat Kernels and Dirac Operators, Grundlehren der Mathematischen Wissenschaften [Fundamental Principles of Mathematical Sciences], 298. Springer-Verlag, Berlin, 1992.

[3] B. Booß-Bavnbek and K. P. Wojciechowski, Elliptic Boundary Problems for Dirac Operators, Mathematics: Theory \& Applications. Birkhäuser Boston Inc., Boston, MA, 1993.

[4] R. Bott, Nondegenerate critical manifolds, Ann. of Math. (2) 60 (1954), 248-261.

[5] P. B. Gilkey, Asymptotic Formulae in Spectral Geometry, Studies in Advanced Mathematics. Chapman \& Hall/CRC, Boca Raton, FL, 2004.

[6] J. Milnor, Morse Theory, Based on lecture notes by M. Spivak and R. Wells. Annals of Mathematics Studies, No. 51. Princeton University Press, Princeton, N.J., 1963.

[7] Lectures on the h-Cobordism Theorem, Notes by L. Siebenmann and J. Sondow. Princeton University Press, Princeton, N.J., 1965. 
[8] J. Roe, Elliptic Operators, Topology and Asymptotic Methods, volume 395 of Pitman Research Notes in Mathematics Series, Longman, Harlow, second edition, 1998.

[9] M. E. Taylor, Partial Differential Equations I, volume 115 of Applied Mathematical Sciences, Springer-Verlag, New York, 1996.

[10] E. Witten, Supersymmetry and Morse theory, J. Differential Geom. 17 (1982), no. 4, 661-692.

[11] W. Zhang, Lectures on Chern-Weil Theory and Witten Deformations, volume 4 of Nankai Tracts in Mathematics, World Scientific Publishing Co. Inc., River Edge, NJ, 2001.

Mathematisches Institut

Georg-August-Universität Göttingen, Germany

AND

Institute for Advanced Studies in Basic Sciences (IASBS)

ZANJAN, IRAN

E-mail address: zadeh@uni-math.gwdg.de 